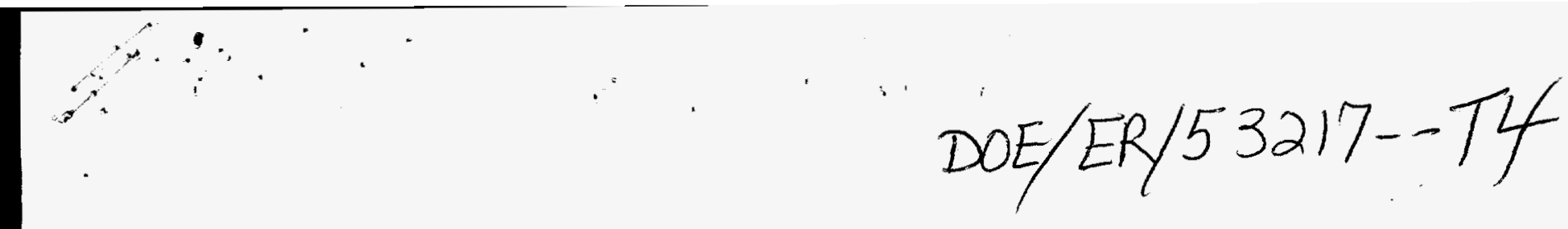

\title{
Theoretical Atomic Physics for Fusion
}

\section{Annual Report}

\author{
Submitted to
}

Office of Fusion Energy

U. S. Department of Energy

Washington, D. C. 20545

by

Michael S. Pindzola

Principal Investigator

Grant No. DE-FG05-86ER53217

Department of Physics

Auburn University

Auburn, AL 36849

Serves

DISTRISUTION OF THIS DOCUMENT IS UALMITED

as final Aepont for DE-FGO5-86ER53217

Renewal out of Chicago Operations

is DE-FG05-96ER 54348

MASTER 


\section{DISCLATMER}

This report was prepared as an account of work sponsored by an agency of the United States Government. Neither the United States Government nor any agency thereof, nor any of their employees, makes any warranty, express or implied, or assumes any legal liability or responsibility for the accuracy, completenese, or usefulness of any information, apparatus, product, or process disclosed, or represents that its use would not infringe privately owned rights. Reference berein to any specific commercial product, process, or service by trade name, trademart, inanufacturet, or otherwise does not necessarily constitute or imply its endorsement, recommendation, or favoring by the United States Govermment or any agency thereof. The views and opinions of authors expressed herein do not secessarily state or reflect those of the United States Government or any agency thereof. 


\section{DISCLAIMER}

Portions of this document may be illegible in electronic image products. Images are produced from the best available original document. 


\section{Introduction}

The understanding of electron-ion collision processes in plasmas remains a key factor in the ultimate development of nuclear fusion as a viable energy source for the nation. Our 1993-1995 research proposal delineated several areas of research in electron-ion scattering theory. In the paragraphs below we summarize our efforts in 1995.

\section{Research}

\section{ELECTRON-IMPACT EXCITATION OF ATOMIC IONS}

\section{A. On the Inclusion of Radiation Damping in the Close-Coupling Equations for Electron-Atom Scattering}

In a paper by Robicheaux et al.(see Appendix 1), an imaginary optical potential was introduced into the R-matrix method to take into account the effect of radiation damping. Of special note is the section on connections to other methods which contains subsections on (1) Fano profiles (2) Feshbach projection operator methods, (3) Davies and Seaton time-dependent methods, and (4) multi- channel quantum defect theory. This long paper on the general theory will form the basis for a number of applications in electron scattering and photoionization of atomic ions.

B. Radiative Damping and Interference in the Resonance Structure of the Electron-Impact Excitation Cross Sections for $\mathbf{T i}^{20+}$

In a paper by Gorczyca et al.(see Appendix 2), a Breit-Pauli R-matrix closecoupling method was employed to calculate various excitation cross sections in 
He-like Ti. They are the first R-matrix calculations for electron-ion excitation in which we employed an optical potential for the radiative damping of intermediate resonance states. The radiation-damped calculations were found to be in reasonable agreement with measurements made at Livermore's EBIT [1].

\section{Resonance Enhancement of Electron-Projectile Excitation in Collisions of $\mathrm{O}^{5+}$ Ions with $\mathrm{H}_{2}$ Targets}

In a paper by Gorczyca and Pindzola (see Appendix 3), an LS R-matrix closecoupling method was used to calculate the $1 s^{2} 2 s\left({ }^{2} S\right) \rightarrow 1 s 2 s 2 p\left({ }^{4} P\right)$ excitation cross section in $\mathrm{O}^{5+}$. Resonance contributions were found to be important. When convoluted with the Compton profile for $\mathrm{H}_{2}$, so that comparisons with ion-molecule experiments could be made, the resonance enhancements were washed out. Thus, the current ion-atom or ion-molecule experiments shed little light on the resonance scattering dynamics found in the near threshold energy region.

\section{Electron-Impact Excitation and Ionization of $\mathrm{Cu}^{+}$}

In a paper by Griffin and Pindzola (see Appendix 4), an LS R-matrix closecoupling method was used to calculate excitation cross sections from $3 d^{10}$ to the terms of the $3 d^{9} 4 s$ and $3 d^{9} 4 p$ configurations of $\mathrm{Cu}^{+}$. The cross sections to the metastable terms $3 d^{9} 4 s^{3} D$ and $3 d^{9} 4 s^{1} D$ were found to be dominated by resonant contributions. Polarization effects reduced the dipole-allowed excitation of the $3 d^{9} 4 p^{1} P$ term by about 27 percent. 


\section{ELECTRON-IMPACT IONIZATION OF ATOMIC IONS}

\section{A. Electron-Impact Ionization of Na-like Ions}

In support of ongoing electron ionization experiments at the Test Storage Ring near Heidelberg, Germany by Professor A Muller and his collaborators, we carried out a series of distorted-wave calculations for the Na-like ions $\mathrm{Cl}^{6+}, \mathrm{Fe}^{15+}$, and $\mathrm{Se}^{23+}$. The intermediate-coupled distorted-wave calculations are in substantial agreement with the magnitude and the general appearance of the measured cross sections for $\mathrm{Fe}^{15+}$, but do not reproduce all details of the rich structure seen in the experiment [2]. Work on $\mathrm{Cl}^{6+}$ using the radiation-damped $\mathrm{R}$-matrix codes is in progress.

\section{B. Electron-Impact Ionization of Ions in the Mo Isonuclear Sequence}

In a paper by Bannister et al.(see Appendix 5), a configuration-average distorted-wave method was used to calculate ionization cross sections from the ground states of $\mathrm{Mo}^{4+}$ and $\mathrm{Mo}^{5+}$. The measured cross sections are in good agreement with theory and are dominated by contributions from excitationautoionization. We plan to work with Salzborn's group at the University of Giessen in comparing theory with experiment for $\mathrm{Mo}^{+}$through $\mathrm{Mo}^{8+}$. The $\mathrm{Mo}$ ionization project is in response to the needs of the MIT plasma modeling group.

\section{Electron-Impact Ionization of Impurity Ions for ADAS}

In support of the JET collisional-radiative codes and associated atomic data bank (ADAS), the distorted-wave method was used to generate ionization cross sections along three different lines. First, configuration-average direct ionization 
codes were modified to produce LS term to term rate coefficients for the light impurity ions ( $\mathrm{C}$ and $\mathrm{O})$. Second, our general close-coupling/distorted-wave codes were modified to produce LS term resolved excitation-autoionization contributions for the transition metal ions (Ti). Third, general distorted-wave direct ionization codes were used to calculate LS term resolved cross sections for neutral $\mathrm{Cr}, \mathrm{Fe}$, $\mathrm{Cu}$, and $\mathrm{Mo}$. These are needed to investigate impurity influx from divertor plates in current and planned tokamaks.

\section{ELECTRON-IMPACT RECOMBINATION OF ATOMIC IONS}

\section{A. Interference between Radiative and Dielectronic Recombination for Highly-Charged Ions}

In a paper by Pindzola et al. (see Appendix 6), a fully-relativistic distortedwave method was used to calculate partial photorecombination cross sections for $\mathrm{U}^{90+}$ and $\mathrm{U}^{88+}$. Interference between the radiative and dielectronic recombination processes was found to be more important for the weaker x-ray resolved partial cross sections than for the total cross sections. This confirms the possibility that recent asymmetries seen in the $\mathrm{x}$-ray resolved photorecombination spectra from a mixture of highly-charged uranium ions [3] may be due to quantum interference.

\section{Activities}

\section{PERSONNEL}

Professor Michael S. Pindzola (PI) is supported on the grant for the summer of 1995. Dr. Thomas W. Gorczyca is supported as a Research Fellow on the grant from January 1, 1995 to August 15, 1995. This Fall Dr. Gorczyca will 
start as an Assistant Professor at the University of Western Michigan. Dr. John A. Shaw, who just finished his Ph.D at the College of William and Mary with Professor John Delos, will be supported as a Research Fellow on the grant beginning September 15, 1995. Professor Francis J. Robicheaux, who is supported by NSF through a prestigious Young Investigator Award, actively participates in our research activities. Two doctoral students are supported by NSF in our small theory group, Mr. P. Gavras and Mr. C. T. Chen. We work extensively with Professor Donald C. Griffin at Rollins College, who is an Adjunct Professor of Physics at Auburn, and with Professor Carolyn L. McCreary and her students in the Department of Computer Sciences and Engineering at Auburn. Through a consulting agreement with JET and a NATO travel grant we collaborate with Dr. Nigel R. Badnell and Professor Hugh P. Summers at the University of Strathclyde. Through a consulting agreement with ORNL we collaborate with Dr. David R. Schultz and Professor Joseph H. Macek in the Physics Division. Through a German government travel grant, Mr. M. Zimmermann, a doctoral student at the University of Giessen under Professor Werner Scheid, visited us last Fall for three months.

\section{PUBLICATIONS AND CONFERENCE ABSTRACTS}

All research publications and conference abstracts for 1994-1995 are listed in Appendix 7.

\section{COMPUTING}

The bulk of our computational work was done on the CRAY2 and CRAYC90 supercomputers at the National Energy Research Supercomputer Center in Livermore, California, the Intel Paragon supercomputers at the High Performance 
Computing Research Center in Oak Ridge, Tennessee, the CRAYC90 supercomputer at the Alabama Supercomputer Center in Huntsville, Alabama, the Sun Sparc workstations in the Department of Physics at Auburn, a 32-node Ncube in the Department of Computer Science at Auburn, and a 128-node Intel PSC 860 in the Engineering and Mathematics Division at ORNL. 


\section{REFERENCES}

[1] "Measurement of Electron Impact Excitation Cross Sections for Heliumlike Titanium,"

S Chantrenne, P Beiersdorfer, R Cauble, and M B Schneider, Phys. Rev. Lett. 69, 265 (1992).

[2] "Electron-Impact Ionization of $\mathrm{Fe}^{15+}$ Ions: An Ion Storage Ring Cross Section Measurement,"

J Linkemann, A Muller, J Kenntner, D Habs, D Schwalm, A Wolf, N R Badnell, and M S Pindzola, Phys. Rev. Lett. 74, 4173 (1995).

[3] "Observation of Interference between Dielectronic Recombination and Radiative Recombination in Highly Charged Uranium Ions,"

D A Knapp, P Beiersdorfer, M H Chen, J H Scofield, and D Schneider, Phys. Rev. Lett. 74, 54 (1995). 


\title{
On the inclusion of radiation damping in the close-coupling equations for electron-atom scattering

F. Robicheaux, T. W. Gorczyca, and M. S. Pindzola

Department of Physics, Auburn University, Auburn, AL 36849

\author{
N. R. Badnell \\ Department of Physics and Applied Physics, University of Strathclyde, Glasgow, G4 ONG, U.K.
}

(October 19, 1994)

\begin{abstract}
The close-coupling approximation for electron-atom scattering is extended to include the effect of one-photon radiation damping at a level that is nonperturbative in the wave function. The complex potential is derived and introduced directly into the set of coupled integro-differential equations used to calculate the S-matrix. The formulation is ideal for inelastic scattering and photoionization with radiation damping and can be used to calculate photorecombination cross sections. The numerical solution of the resulting differential equation is accomplished through a combination of R-matrix, perturbation theory, and analytic techniques. Some of the implications of this method are discussed. The connections to previous theoretical approaches are discussed.
\end{abstract}

34.80.Kw, 31.30.Jv, 31.10.+z 


\title{
Radiative Damping and Interference in the Resonance Structure of the Electron-Impact Excitation Cross Sections for $\mathbf{T i}^{20+}$ 1995
}

\author{
T. W. Gorczyca, F. Robicheaux, and M. S. Pindzola \\ Department of Physics, Auburn University, Auburn, AL 36849 \\ N. R. Badnell \\ Department of Physics and Applied Physics, University of Strathclyde, Glasgow, G4 ONG, UK
}

(March 28, 1995)

\begin{abstract}
We have performed the first R-matrix calculations for the electron-impact excitation of a highly-charged ion in which an optical potential is included to allow for radiative damping of intermediate resonance states. The present case considered is that of electrons incident on $\mathrm{Ti}^{20+}$ ions. Comparisons with undamped R-matrix calculations, distorted-wave calculations, and experimental measurement are made. The radiative optical potential accounts for significant damping of high-n resonances. Configuration-interaction between resonances, which is automatically incorporated in the R-matrix method but usually omitted in the implementation of distorted-wave techniques, was found to increase the resonance contributions considerably. Our radiative-damped R-matrix cross sections show fairly good agreement with the resonance profile obtained from Electron Beam Ion Trap measurements [S. Chantrenne et al., Phys. Rev. Lett. 69, 265 (1992)]. This system demonstrates the necessity for simultaneously including radiative-damping and interfering-resonance effects
\end{abstract}


within electron-highly-charged ion scattering calculations.

34.80.Kw

Typeset using REVTEX 


\title{
Resonance Enhancement of Electron-Projectile Excitation in Collisions of $\mathrm{O}^{5+}$ Ions with $\mathrm{H}_{2}$ Targets
}

\author{
T. W. Gorczyca and M. S. Pindzola \\ Department of Physics, Auburn University, Auburn, AL 36849
}

(February 13, 1995)

\begin{abstract}
The electron-impact excitation cross section for the $1 s^{2} 2 s\left({ }^{2} S\right) \rightarrow 1 s 2 s 2 p\left({ }^{4} P\right)$ transition in $\mathrm{O}^{5+}$ has been calculated using the $\mathrm{R}$-matrix method. Resonance contributions are found to increase the background cross section by factors of 2-5 in the $550-700 \mathrm{eV}$ energy region when convoluted with a $1 \mathrm{eV} \mathrm{FWHM}$ Gaussian profile. When convoluted with the Compton profile for $\mathrm{H}_{2}$, however, the difference between the background and resonance enhanced cross sections is less than $5 \%$ near the peak. Thus, experiments involving collisions of $\mathrm{O}^{5+}$ ions with $\mathrm{H}_{2}$ targets shed little light on the resonance scattering dynamics found in the near threshold energy region.

$34.80 . \mathrm{Kw}$
\end{abstract}

Typeset using REVTEX 


\title{
Absolute cross sections for electron-impact single ionization of $\mathrm{Mo}^{4+}$ and $\mathrm{Mo}^{5+}$ ions

M. E. Bannister and F. W. Meyer

Physics Division, Oak Ridge National Laboratory, Oak Ridge, Tennessee 38731-6372

Y. S. Chung, N. Djurić, and G. H. Dunn *

Joint Institute for Laboratory Astrophysics of the University of Colorado

and the National Institute of Standards and Technology, Boulder, Colorado 80309-0440

M. S. Pindzola

Department of Physics, Auburn University, Auburn, Alabama 36849

D. C. Griffin

Department of Physics, Rollins College, Winter Park, Florida 32789

(Received January 17, 1995)

\begin{abstract}
Absolute total cross sections for electron-impact single ionization of $\mathrm{Mo}^{4+}$ and $\mathrm{Mo}^{5+}$ ions have been measured using a crossed-beams technique from below the ground-state threshold to $500 \mathrm{eV}$ with typical absolute uncertainties of $9 \%$ near the peak of the cross sections. The measured cross sections are in good agreement with distorted-wave calculations and are dominated by contributions from excitation autoionization. Nonzero cross sections below the threshold for ionization of $\mathrm{Mo}^{5+}\left(4 p^{6} 4 d^{2}\right)$ ground-state ions indicate
\end{abstract}

\footnotetext{
"Quantum Physics Division, National Institute of Standards and Technology
} 
that metastable ions were present in the beam extracted from an electroncyclotron-resonance ion source. No evidence of metastables was found in the case of the $\mathrm{Mo}^{4+}$ measurements. Molybdenum ion production in the source was facilitated by a mini-oven sublimating $\mathrm{MoO}_{3}$.

PACS number(s): $34.80 . \mathrm{Kw}$

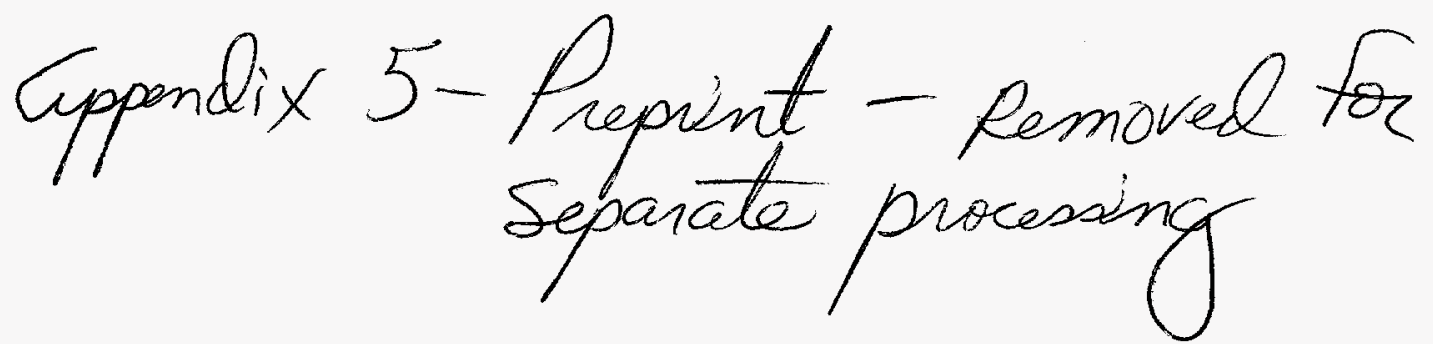


Append ix 6

\title{
Photorecombination of Highly Charged Uranium Ions
}

M. S. Pindzola and F. J. Robicheaux

Department of Physics, Auburn University, Auburn. AL 36849

N. R. Badnell

Department of Physics, University of Strathclyde, Glasgow, Scotland G4 ONG

M. H. Chen

Department of Physics, Lawrence Livermore National Laboratory, Livermore, CA

M. Zimmermann

Department of Physics, University of Giessen, Giessen, Germany

(March 28, 1995)

\begin{abstract}
Photorecombination cross sections for highly charged uranium ions are calculated using many-body perturbation theory. The cross sections are resolved as to the final recombined level, corresponding to different $\mathrm{x}$-ray light emitted during radiative stabilization of the dielectronic capture resonances. Interference between the radiative and dielectronic recombination processes is found to be more important for the weaker $x$-ray resolved partial photorecombination cross sections than for the total cross sections.
\end{abstract}




\section{Appendix 7}

\section{Letters and Articles in the American Physical Review}

1. "Dielectronic Capture Processes in the Electron-Impact Ionization of $\mathrm{Sc}^{2+}$," M.S. Pindzola, T.W. Gorczyca, N.R. Badnell, D.C. Griffin, M. Stenke, G. Hofmann, B. Weissbecker, K. Tinschert, E. Salzborn, A. Müller, G.H. Dunn, PRA 49, 933 (1994).

2. "Excitation-Autoionization Contributions to the Electron-Impact Ionization of $\mathrm{Kr}^{4+}$ $\mathrm{Kr}^{7+}, "$

T.W. Gorczyca, M.S. Pindzola, D.C. Griffin and N.R. Badnell, PRA 49, 4682 (1994).

3. "The Nature of Interacting Resonances in Electron-Ion Excitation,"

D.C. Griffin, M.S. Pindzola, F. Robicheaux, T.W. Gorczyca and N.R. Badnell, PRL 72, 3491 (1994).

4. "Interacting Resonance Effects along an Isoelectronic Sequence: Electron-Impact Excitation of Mg-like Ions,"

N.R. Badnell, D.C. Griffin, T.W. Gorczyca and M.S. Pindzola, PRA 50, 1231 (1994).

5. "Time-dependent Approach to Atomic Autoionization,"

D.R. Schultz, C. Bottcher, D.H. Madison, J.L. Peacher, G. Buffington, M.S. Pindzola, T.W. Gorczyca, P. Gavras and D.C. Griffin, PRA 50, 1348 (1994).

6. "Resonance Structure in the $4 s^{2}\left({ }^{1} S\right) \rightarrow 4 s 4 p\left({ }^{3} P_{0,1,2}\right)$ Electron-Impact Excitation of $\mathrm{Kr}^{6+}, "$

T.W. Gorczyca, M.S. Pindzola, N.R. Badnell, and D.C. Griffin, PRA 51, 488 (1995).

7. "Electron-Impact Ionization of the Fe Atom,"

M.S. Pindzola, D.C. Griffin, and J.H. Macek, PRA 51, 2186 (1995).

8. "Elastic and Inelastic Scattering of Electrons from $\mathrm{Ar}$ and $\mathrm{Cl}$,"

D.C. Griffin, M.S. Pindzola, and T.W. Gorczyca, PRA 51, 2265 (1995).

9. "Electron-Impact Ionization of $\mathrm{Fe}^{15+}$ Ions,"

J. Linkemann, A. Muller, J. Kenntner, D. Habs, D. Schwalm, A. Wolf, N. R. Badnell, and M. S. Pindzola, PRL 74, 4173 (1995).

10. "Time-Dependent Unrestricted Hartree-Fock Theory for the Multiphoton Ionization of Atoms,"

M. S. Pindzola, P. Gavras, and T. W. Gorczyca, PRA 51, 3999 (1995).

11. "On the Inclusion of Radiation Damping in the Close-Coupling Equations for ElectronAtom Scattering,"

F. Robicheaux, T.W. Gorczyca, M.S. Pindzola,, and N.R. Badnell, accepted for publication.

12. "Photorecombination of Highly Charged Uranium Ions,"

M. S. Pindzola, F. J. Robicheaux, N. R. Badnell, M. H. Chen, and M. Zimmermann, accepted for publication. 
13. "Absolute Cross Sections for Electron-Impact Single Ionization of $\mathrm{Mo}^{4+}$ and $\mathrm{Mo}^{5+}$ Ions",

M. E. Bannister, F. W. Meyer, Y. S. Chung, N. Djuric, G. H. Dunn, M. S. Pindzola, and D. C. Griffin, accepted for publication.

14. "Resonance Enhancement of Electron-Projectile Excitation in Collisions of $\mathrm{O}^{5+}$ Ions with $\mathrm{H}_{2}$ Targets",

T. W. Gorczyca and M. S. Pindzola, accepted for publication.

15. "High Resolution Measurement of Dielectronic Recombination of Fluorine- like Selenium Ions,"

A. Lampert, D. Habs, G. Kilgus, D. Schwalm, A. Wolf, M.S. Pindzola and N.R. Badnell, submitted for publication.

16. 'Direct Solution of the Time-Dependent Schrodinger Equation for Proton - Hydrogen Collisions in 2D Flatland,"

P. Gavras, M. S. Pindzola, D. R. Schultz, and J. C. Wells, submitted for publication.

17. "Radiative Damping and Interference in the Resonance Structure of the ElectronImpact Excitation Cross Sections for $\mathrm{Ti}^{20+} "$,

T. W. Gorczyca, F. Robicheaux, M. S. Pindzola, and N. R. Badnell, submitted for publication.

18. "Elimination of Electron-Ion Pseudoresonances within the R-matrix Method," T. W. Gorczyca, F. Robicheaux, M. S. Pindzola, N. R. Badnell, and D. C. Griffin, submitted for publication.

\section{Letters and Articles in the Journal of Physics (London)}

1. "Configuration-Interaction Effects on the Electron-Impact Ionization of $\mathrm{Ti}^{3+}$, T.W. Gorczyca, M.S. Pindzola, D.C. Griffin and N.R. Badnell, JPB 27, 2399 (1994).

2. 'Mixed Gauge Approach to Multiphoton Wavefunctions," F. Robicheaux, C. T. Chen, P. Gavras, and M. S. Pindzola, accepted for publication.

3. "Excitation and Ionisation of Neutral $\mathrm{Cr}$ and Mo and the Application to Impurity Influx,"

N.R. Badnell, T.W. Gorczyca, M.S. Pindzola, and H.P. Summers, submitted for publication.

4. "Electron-Impact Excitation and Ionization of $\mathrm{Cu}^{+}, "$

D. C. Griffin and M. S. Pindzola, submitted for publication.

\section{Letters and Articles in Other Journals}

1. "Dielectronic Recombination of Lithium-like $\mathrm{Ar}^{15+}$,

S. Schennach, A. Müller, O. Uwira, J. Haselbauer, W. Spies, A. Frank, M. Wagner, R. Becker, M. Kleinod, E. Jennewein, N. Angert, P.H. Mokler, N.R. Badnell and M.S. Pindzola, Z. Phys. D 30, 291 (1994). 
2. "Photoionization of a Model Helium Atom," M. S. Pindzola, P. Gavras, and T. W. Gorczyca, in Few-Body Problems in Physics, ed. Franz Gross, (AIP, New York, 1995), p. 667.

3. "Adaptation of Asymptotic Close-Coupling Methods to Massively Parallel Computers,"

T.W. Gorczyca, M.S. Pindzola, F.S. Shieh and C.L. McCreary, accepted for publication to Comput. Phys. Commun.

\section{Review Articles in Books and Journals}

1. "Density and Field Effects on Dielectronic Recombination in a Generalized CollisionalRadiative Model,"

N.R. Badnell, W.J. Dickson, D.C. Griffin, M.S. Pindzola, and H.P. Summers, in Atomic Processes in Plasmas, (AIP, San Antonio, 1995), p.84.

2. "Electron-Ion Collisions using Close-coupling and Distorted-Wave Theory," D.C. Griffin and M.S. Pindzola in Many-Body Atomic Physics eds. J.J. Boyle and M.S. Pindzola (Cambridge, 1995).

3. "Dielectronic Recombination,"

M.S. Pindzola, D.C. Griffin, and N.R. Badnell, in Atomic, Molecular and Optical Physics Reference Book, ed. G.W.F. Drake, (AIP, 1995).

4. "Excitation of Atomic Ions by Electron-Impact,"

S.S. Tayal, A.K. Pradhan, and M.S. Pindzola, in Atomic Processes in Magnetically Confined Fusion Edge Plasmas, ed. R.K. Janev, (Plenum, 1995).

5. "Direct Solution of the Time-dependent Schrodinger Equation for Atomic Processes," M.S. Pindzola and P. Gavras, in Atomic Collisions Symposium in Memory of Chris Bottcher, (AIP, 1995).

6. "The Theory of Indirect Processes in Electron-Impact Excitation,"

D.C. Griffin, M.S. Pindzola, T.W. Gorczyca, and N.R. Badnell, in Atomic Collisions Symposium in Memory of Chris Bottcher, (AIP, 1995).

\section{Edited Books}

1. Many-Body Atomic Physics: Lectures on the Application of Many-Body Theory to Atomic Physics in Memory of Professor Hugh P. Kelly,

J. J. Boyle and M. S. Pindzola, (Cambridge Press, 1995).

2. New Developments in Electron-Atom Scattering Theory,

D. C. Griffin and M. S. Pindzola, (AIP Press, 1997), in preparation. 


\section{Invited Talks at Conferences and Workshops}

1. "Direct Solution of the Time-Dependent Schrodinger Equation for Atomic Processes," M. S. Pindzola, International Symposium on Atomic Collisions, March 3-5, 1994, Oak Ridge, Tennessee.

2. "Electron Scattering from Atoms and Ions: Generation of an Atomic Data Base for Fusion,"

M. S. Pindzola and D. C. Griffin, U.S. DOE Workshop on Atomic Physics and Plasma Modeling of Edge/Divertor Plasmas, Oct.28-29, 1994, Oak Ridge, Tennessee.

3. "Double Ionization of Two-Electron Model Atoms,"

M. S. Pindzola and P. Gavras, NSF Workshop on Multi-Electron Atoms in Intense Laser Fields, October 19-21, 1995, Cambridge, Massachusetts.

\section{Invited Colloquiums and Seminars}

1. "Theories of Everything and Atomic Collision Physics,"

M. S. Pindzola, LLNL Physics Division Seminar, Jan. 1994, Livermore, California.

2. "Review of ORNL Theoretical Atomic Physics for Fusion Program," M. S. Pindzola, U.S. DOE Review Committee Meeting, April 1994, Oak Ridge, Tennessee.

3. "Photoionization and Autoionization of a Model Helium Atom,"

M. S. Pindzola, Harvard University Physics Department Colloquium, Nov. 1994, Cambridge, Massachusetts.

\section{Contributed Conference Abstracts}

1. "On the Nature of Interacting Resonances in Electron-Impact Excitation,"

D.C. Griffin, M.S. Pindzola, F. Robicheaux, T.W. Gorczyca, and N.R. Badnell, APS Division of Atomic, Molecular and Optical Physics Meeting, April 18-21, 1994, Washington, D.C.

2. "Electron-Impact Excitation Cross Section for $\mathrm{Kr}^{6+}$,"

T.W. Gorczyca, M.S. Pindzola, N.R. Badnell and D.C. Griffin, APS Division of Atomic, Molecular and Optical Physics Meeting, April 18-21, 1994, Washington, D.C.

3. "Ionization of Two-Electron Model Atoms,"

P. Gavras, M.S. Pindzola, T.W. Gorczyca, and D.C. Griffin APS Division of Atomic, Molecular and Optical Physics Meeting, April 18-21, 1994, Washington, D.C.

4. "Photoionization of a Model Helium Atom,"

M. S. Pindzola, P. Gavras, and T. W. Gorczyca, XIV International Conference on Few Body Problems in Physics, May 26-31, 1994, Williamsburg, Virginia. 
5. "Elastic and Inelastic Scattering from $\mathrm{Ar}$ and $\mathrm{Cl}$,"

D.C. Griffin, M.S. Pindzola, T.W. Gorczyca, and N.R. Badnell, XIV International Conference on Atomic Physics, July 31-August 5, 1994, Boulder, CO.

6. "Electron-Impact Excitation and Ionization Cross Sections for Cr and Mo," T.W. Gorczyca, M.S. Pindzola, N.R. Badnell, H.P. Summers, and D.C. Griffin, XIV International Conference on Atomic Physics, July 31-August 5, 1994, Boulder, CO.

7. "Unified and Independent Processes Recombination Cross Sections," M.S. Pindzola, M.H. Chen and N.R. Badnell, VII International Conference on the Physics of Highly Charged Ions, September 19-23, 1994, Vienna, Austria.

8. "Resonant Electron Impact Ionization and Recombination of Li-like $\mathrm{Cl}^{14+}$ and $\mathrm{Si}^{11+}$ at the Heidelberg Test Storage Ring,"

J. Kenntner, J. Linkemann, N.R. Badnell, C. Broude, D. Habs, G. Hoffmann, A. Muller, M.S. Pindzola, E. Salzborn, D. Schwalm, and A. Wolf, VII International Conference on the Physics of Highly Charged Ions, September 19-23, 1994, Vienna, Austria.

9. "Dielectronic Recombination of Na-like Iron,"

J. Linkemann, J. Kenntner, A. Muller, A. Wolf, D. Habs, D. Schwalm, W. Spies, O. Uwira, A. Frank, M.S. Pindzola, and N.R. Badnell, VII International Conference on the Physics of Highly Charged Ions, September 19-23, 1994, Vienna, Austria.

10. "Electron-Impact Ionization of Sodium-like $\mathrm{Fe}^{15+}$ Ions,"

J. Linkemann, J. Kenntner, A. Muller, A. Wolf, D. Habs, D. Schwalm, W. Spies, O. Uwira, A. Frank, A. Liedtke, G. Hofmann, E. Salzborn, M.S. Pindzola, and N.R. Badnell, VII International Conference on the Physics of Highly Charged Ions, September 19-23, 1994, Vienna, Austria.

11. 'Dielectronic Recombination of Very-Heavy Highly-Charged Ions,"

M. Zimmermann, K. Kollmar, N. Grun, W. Scheid, and M. S. Pindzola, V European Conference on Atomic and Molecular Physics, April 3-7, 1995, Edinburgh, Scotland.

12. 'Resonance Structures due to Dielectronic Capture Processes in the Electron-Impact Ionization of $\mathrm{Li}$ and $\mathrm{Na}$ like Ions,"

M. S. Pindzola and N. R. Badnell, V. European Conference on Atomic and Molecular Physics, April 3-7, 1995, Edinburgh, Scotland.

13. 'Excitation and Ionization of Neutral $\mathrm{Cr}$ and $\mathrm{Mo}$ and the Application to Impurity Influx,"

N. R. Badnell, T. W. Gorczyca, M. S. Pindzola, and H. P. Summers, V European Conference on Atomic and Molecular Physics, April 3-7, 1995, Edinburgh, Scotland.

14. "Electron-Impact Ionization and Dielectronic Recombination of Na-like Ions," J. Linkemann, J. Kenntner, A. Muller, A. Wolf, D. Habs, D. Schwalm, W. Spies, O. Uwira, A. Frank, A. Liedtke, G. Hofmann, E. Salzborn, M. S. Pindzola, and N. R. Badnell, V European Conference on Atomic and Molecular Physics, April 3-7, 1995, Edinburgh, Scotland. 
15. "Electron-Impact Ionization and Dielectronic Recombination of Highly Charged Lithiumlike Ions in an Ion Storage Ring,"

J. Kenntner, D. Habs, D. Schwalm, A. Wolf, J. Linkemann, A. Muller, N. R. Badnell and M. S. Pindzola, V European Conference on Atomic and Molecular Physics, April 3-7, 1995, Edinburgh, Scotland.

16. "Radiation-Damped R-Matrix Calculations for the Electron-Impact Excitation of $\mathrm{Ti}^{20+}$ ", T. W. Gorczyca, F. J. Robicheaux, and M. S. Pindzola, APS Division of Atomic, Molecular, and Optical Physics Meeting, May 17-19, 1995, Toronto, Ontario.

17. "Time-Dependent Proton-Hydrogen Collisions in Flatland", P. Gavras and M. S. Pindzola, APS Division of Atomic, Molecular, and Optical Physics Meeting, May 17-19, 1995, Toronto, Ontario.

18. "Final-State Correlation Effects in the Electron-Impact Ionization of Complex Atoms", M. S. Pindzola and D. C. Griffin, APS Division of Atomic, Molecular, and Optical Physics Meeting, May 17-19, 1995, Toronto, Ontario.

19. "Electron-Impact Single Ionization of $\mathrm{Mo}^{4+}$ and $\mathrm{Mo}^{5+}$," M. E. Bannister, F. W. Meyer, Y. S. Chung, N. Djuric, G. H. Dunn, M. S. Pindzola, and D. C. Griffin, APS Division of Atomic, Molecular, and Optical Physics Meeting, May 17-19, 1995, Toronto, Ontario. 